\title{
Facial expression recognition using three- stage support vector machines
}

\author{
Issam Dagher ${ }^{*}$, Elio Dahdah and Morshed Al Shakik
}

\begin{abstract}
Herein, a three-stage support vector machine (SVM) for facial expression recognition is proposed. The first stage comprises 21 SVMs, which are all the binary combinations of seven expressions. If one expression is dominant, then the first stage will suffice; if two are dominant, then the second stage is used; and, if three are dominant, the third stage is used. These multilevel stages help reduce the possibility of experiencing an error as much as possible. Different image preprocessing stages are used to ensure that the features attained from the face detected have a meaningful and proper contribution to the classification stage. Facial expressions are created as a result of muscle movements on the face. These subtle movements are detected by the histogram-oriented gradient feature, because it is sensitive to the shapes of objects. The features attained are then used to train the three-stage SVM. Two different validation methods were used: the leave-one-out and K-fold tests. Experimental results on three databases (Japanese Female Facial Expression, Extended Cohn-Kanade Dataset, and Radboud Faces Database) show that the proposed system is competitive and has better performance compared with other works.
\end{abstract}

Keywords: Facial expression recognition, Support vector machine, Histogram of oriented gradients, Viola-Jones, Validation

\section{Introduction}

Artificial intelligence has created higher standards for innovation and has introduced new possibilities for human-computer interaction. The goal of achieving communication between computers and humans is now a possibility; however, $55 \%-94 \%$ of human communication is nonverbal [1]. Thus, there is a need to develop an accurate facial expression recognition (FER) system that can establish efficient communication between humans and computers. The development of such a system can also be useful in several areas, such as lie detectors, surveillance, smart computing, visual development, computer gaming, and augmented reality [2].

For efficient communication to occur between two people, seven expressions are globally identified and analyzed to make communication smooth and reliable. Translating this idea into a computer system, the FER system can improve the way computers interact with humans and lead to further advancements in this field.

\footnotetext{
*Correspondence: dagheri@hotmail.com; jamalhassanie@gmail.com Computer Engineering Department, University of Balamand, Tripoli P.O.BOX 100, Lebanon
}

Many researchers have tried developing the optimal FER system. Generally, the main approaches were to do it via machine learning or deep learning. The majority of studies used the machine-learning approach, because deep learning is a recent trend. Also, deep learning requires a massive amount of computation time, speed, and memory, whereas machine learning does not have all these requirements. The possible combinations are endless, because any or all of the following can be changed: face detection, feature extraction, and classification. Some studies in which the method of feature extraction was changed, whereas the detection and classification were kept the same, are as follows. Shan et al. [3] introduced an FER system that can detect facial expressions using a boosted version of local binary patterns (LBPs) and classified it using support vector machines (SVMs). Shih et al. [4] used linear discriminant analysis (LDA) and SVM. Khan performed a LBP-pyramid with SVM classification [1]. Jaffar [1] performed LBP with a Gabor filter. All these methods are based on machine learning, where the face was detected as a whole, and then each model was implemented. Some researchers decided to segment the face rather than take it as a whole and 
changed their extraction method. Chen et al. [5] applied facial segmentation to separate the eyes and mouth from the face before applying the histogram-oriented gradients (HOG) feature and classifying the image using an SVM. Also, Chen et al. [5] used a patch-based Gabor filter after segmenting each component of the face. Others decided to combine several extraction methods and then speed up the computation time. Liu et al. [6] combined LBP and HOG methods for feature extraction; then, they performed principal component analysis (PCA) to minimize the time and speed needed for computation, and, finally, they used SVM classification. However, some decided to implement deep learning rather than continuing to use machine learning. Mollahosseini et al. [7] used a deep-neural-network system. The number of combinations is endless, as can be seen above; however, their accuracies are fairly competitive. Thus, many researchers have decided to modify images by using various preprocessing techniques - spatial orientation, histogram equalization, color conversion, resolution enhancement, and many others.

The technique applied in this study generally revolves around the preprocessing of the image before extracting its features and the stages of classification. The classification is split into three individual stages composed of binary comparisons where the system enters each stage depending on the necessity and the case.

\section{Related work}

In a previous study [8], the authors of that work used histograms of gradients as a feature extraction method. Three-dimensional FER was analyzed in other research [9]. A dictionary-based approach for FER was used [10]. Gabor wavelets and learning vector quantization were used [11]. FER using contourlet transform was done [12]. Exploring shape deformation is done [13]. In other work [14], SVM was used in FER. A convolutional neural network $(\mathrm{CNN})$ was used [15]. In another study [16], the authors of that report used pairwise feature selection in classification. Multiple CNNs were used in other research [17]. Prototype-based modeling for facial expression analysis was implemented [18]. Curvelet transform was used in FER [19]. A guide to recognizing emotions from facial clues was presented [20]. Analysis of FER with occlusions was done in other work [21]. Another study [22] emphasized the line-based caricatures in FER. FER using extended LBP (ELBP) based on covariance matrix transform in Karhunen-Loeve transform was utilized in ref. [23]. Other researchers [24] used 3-D facial feature distances. Automatic FER using features of salient facial patches was demonstrated [25]. Fisher discriminant analysis was evaluated [26]. Other researchers [27] employed segmentation of face regions in FER. In other work [28], a deep fusion CNN was used in FER.
Also, 2dPCA was used [29]. Other researchers [30] used the LBP as a feature extraction method in FER.

\section{Contributions}

- Three-level-network: This network consists of three stages, where the primary and first stage is made up of 21 SVMs, which are all the binary combinations of the seven expressions. If one expression is dominant, then the first stage suffices. If two are dominant, the second stage is needed. If three are dominant, the third stage is used.

- An image preprocessing stage is used to ensure that the features attained from the face detected have a meaningful and proper contribution to the classification stage.

- Experimental results on three databases, Japanese Female Facial Expression (JAFFE), Extended CohnKanade Dataset $(\mathrm{CK}+)$, and Radboud Faces Database (RaFD), show that the proposed system is competitive and has better performance compared with other systems.

\section{General model and mathematical computation}

The skeletal structure of the model is shown in Fig. 1. The images of the datasets are divided into the training set and testing set. The number of images in each set varies depending on what type of validation method is implemented. The images of each set undergo some preprocessing techniques, followed by facial detection and feature extraction. The features are then classified using SVM classification. Once the system is trained, the set to be tested undergoes a similar process to the trained set; however, ultimately, the features extracted from the image are compared with the SVM classifiers, and the result is then obtained.

Two-part model SVM classifiers are designed in such a way that they are made up of three stages (Fig. 2). Each stage represents a unique function to reach the final result. The first stage consists of a network of 21 SVMs. Because there are seven main expressions, each expression is compared with another expression, thus making a unique binary SVM. The total number of possible combinations resulting from this binary comparison is shown in Eq. (1), where selecting two expressions from the total number of expressions makes it possible to determine all possible binary outcomes.

$$
C_{2}^{7}=\frac{7 !}{2 ! 5 !}=21
$$

\section{(Combination 2 out of 7 expressions)}

There are 21 different results from the SVMs shown above, and each expression has its own counter that 


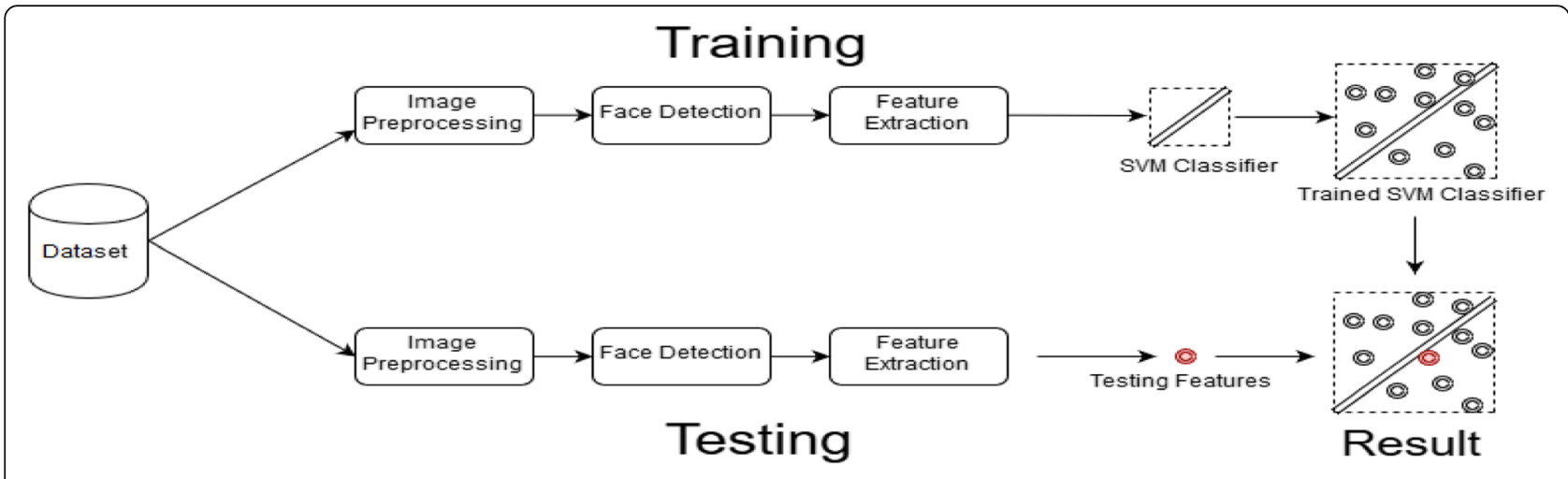

Fig. 1 General skeletal structure: dividing the dataset into training and testing sets

counts the occurrences of the expression at the output of the 21 SVMs. After the occurrences have been calculated, the counters are compared with each other, and the result follows one of the following conditions.

- If one expression occurs the most at the outputs, then the final result definitely represents this expression.

- If two expressions equally occur at the outputs, the two expressions go into another stage, called "Stage 2 ". Stage 2 has one SVM that is trained with these two expressions only and compared one last time to get a final result. The result of the SVM in Stage 2 represents the final result.
- If three expressions occur equally at the outputs, the three expressions enter another stage, called "Stage 3". Similar to Stage 2, Stage 3 has three SVMs that represent all the possible combinations among the three expressions. The same concept of the 21 SVMs is applied, and the occurrences are counted. Then, the final result is determined from the output of this stage.

\section{Datasets and image preprocessing}

To evaluate the performance of the proposed approach, the system was tested on three commonly adopted datasets (Fig. 3): the JAFFE database [31], CK+ [32], and RaFD [33].

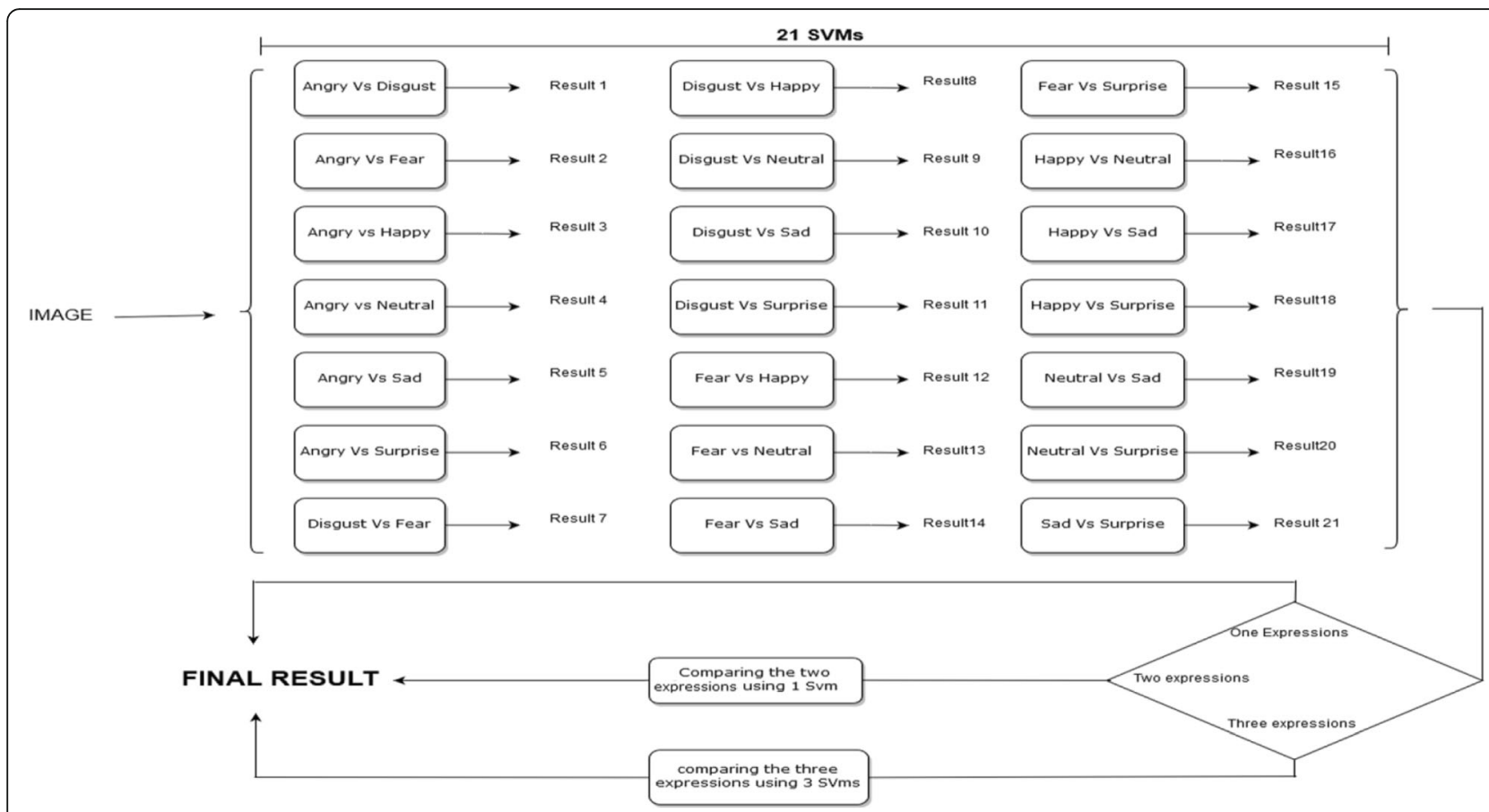

Fig. 2 Two-stage model: first stage (21 SVM) and Second and third stages combining the results of Stage 1 


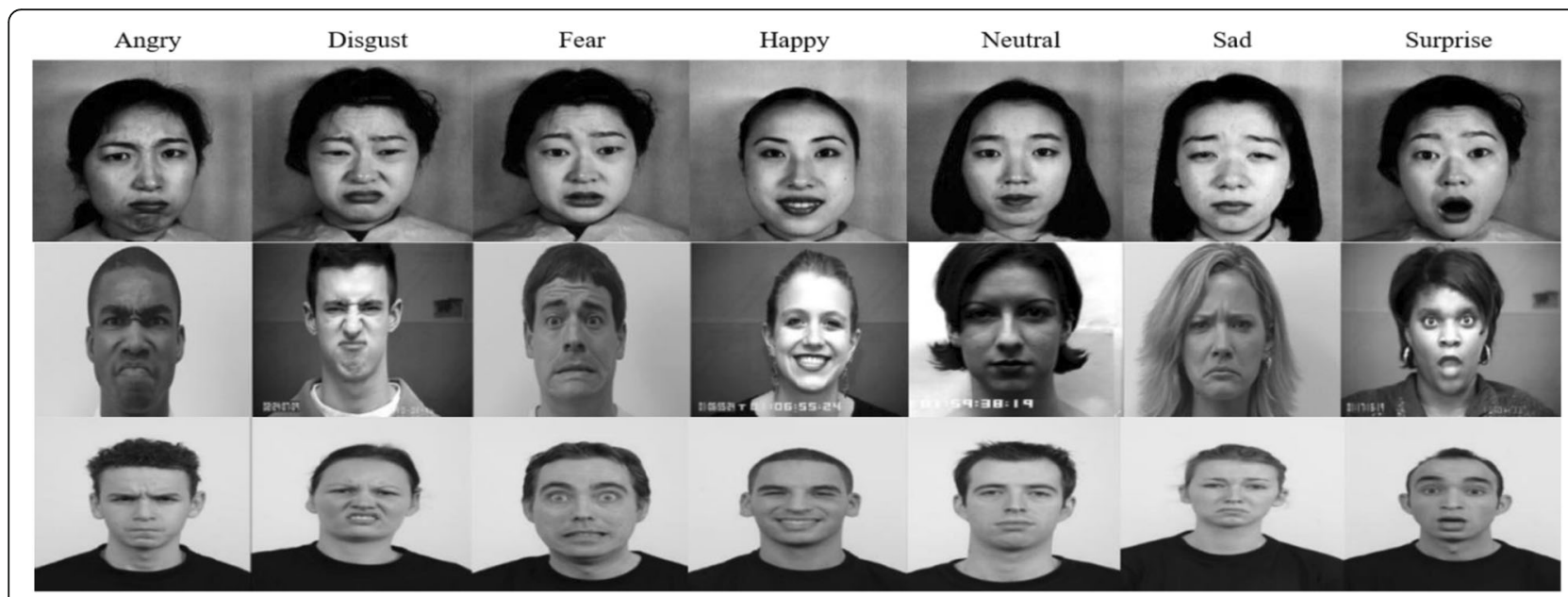

Fig. 3 Sample of seven expressions of each dataset starting from JAFFE (top), CK+ (middle), and RaFD (bottom)

\section{JAFFE database}

The dataset was taken from the Psychology Department at Kyushu University. This database consists of the seven primary expressions that were posed by 10 Japanese female models. The database consists of a total of $213 \mathrm{im}-$ ages, where 30 images are angry, 29 images are disgust, 32 images are fear, 31 images are happy, 30 images are neutral, 31 images are sad, and 30 images are surprise. Each model provided approximately three images for each facial expression. Each image was saved in grayscale with a resolution of $256 \times 256$ [31].

\section{CK+ database}

This dataset consists of eight expressions (seven primary expressions plus contempt) that were posed by more than 200 adults ranging from 18 to 50 years of age. It generally consisted of Euro-American and AfroAmerican individuals. The images were taken in time frames where the initial frame was neutral, which was then transitioned into the expression that was desired at the end frame (the peak frame). These images were saved, some in grayscale and some in color, in $640 \times 490$ or $640 \times 480$ pixels. The database consists of 123 neutral images and 327 peak images (images with a certain expression). These 327 images consist of 45 images that are angry, 18 images that are contempt, 59 images that are disgust, 25 images that are fear, 69 images that are happy, 28 images that are sad, and 83 images that are surprise. The contempt expression images are excluded in this work [32].

\section{RaFD database}

This dataset consists of 67 models: 20 male adults, 19 female adults, 4 male children, 6 female children, and 18 Moroccan male adults. Each model is pictured at a different gaze direction (left, frontal, and right) and at five different camera angles simultaneously. The dataset consists of eight expressions (seven primary expressions + contempt) and 1608 images that are distributed into 201 angry images, 201 contempt images, 201 disgust images, 201 fear images, 201 happy images, 201 neutral images, 201 sad images, and 201 surprise images with a resolution of $640 \times 1024$ pixels each. The frontal facing images (images at an angle of $90^{\circ}$ ) were worked on, and the contempt expression was excluded from this work [33].

\section{Image preprocessing}

The datasets have different properties, such as resolution, size, and color. To unify the system to work on all datasets, standard image properties had to be developed.

As shown in Fig. 4, to reach the standard image input, the following steps were implemented:

- Gray scaling and resizing

- Viola-Jones detection

- Border adjustment

- Cropping

- Additional resizing

\section{Gray scaling and resizing}

The images in the JAFFE dataset were originally in grayscale format, whereas the images in the $\mathrm{CK}+$ and the RaFD datasets had a mixture of color and grayscale images. Therefore, to unify the input type, the images were all tested, whether they were in grayscale format or not. If the images were colored, they were converted to gray. The next step was to ensure that all images were of equal size. The smallest dataset size was the JAFFE dataset, which included images of size $256 \times 256$. Therefore, images of CK+ and RaFD had to be resized to $256 \times 256$ 


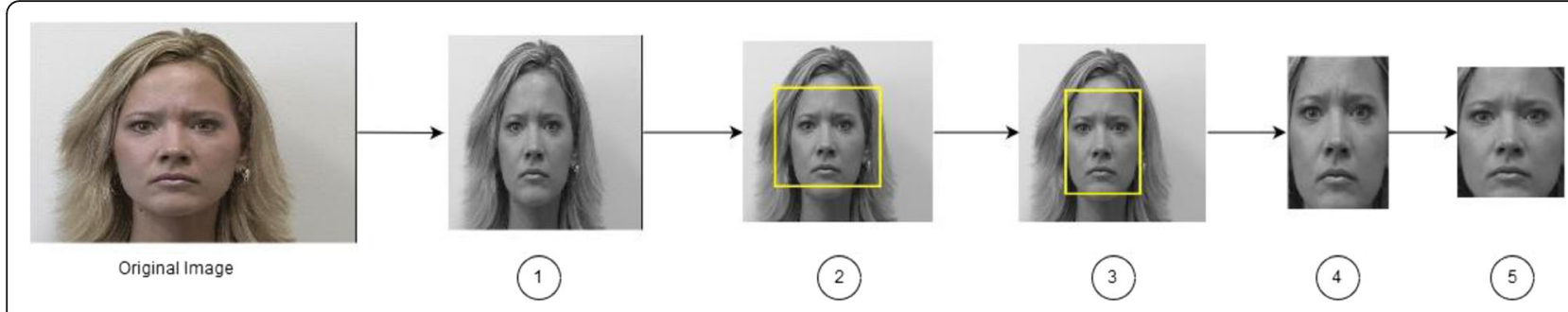

Fig. 4 Image preprocessing: (1) Gray scaling and resizing, (2) Viola-Jones, (3) border adjustment, (4) cropping, and (5) additional resizing• Viola-Jones

to provide a standard input size. Fig. 5 shows the image after performing the grayscale conversion and the resizing. The resolution was not affected, and this not only unified the input size and color but also removed any color lamination that the image may have had.

\section{Viola-Jones detection and border adjustment}

The Viola-Jones algorithm was implemented to detect the face from the image. Some modifications were performed to detect only one face from the image that is the clearest face. This was done by comparing the sizes of the "supposed" faces detected and then selecting the face with the largest dimensions. The Viola-Jones algorithm could capture the majority of the faces of the datasets accurately; however, it experienced some problems detecting faces displaying the surprise expression. The nature of the surprise expression is for the mouth to be wide open with raised eyebrows. As can be seen in Fig. 6 (left), the mouth is cropped, which leads to false classification in the system. Also, extra components were detected at the edges of the face, which also leads to false classifications.

\section{Border adjustment}

Border adjustment was a reasonable solution to these problems. Fig. 6 (right) displays the possible three borders that must be varied to give proper results. To optimize the input image, Border 3 must cover the whole mouth only, whereas Borders 1 and 2 must cover the face region only, without the ears. The optimal value for the extension of Border 3 is 8 pixels downward. Values smaller than this did not solve the problem of the cropped mouth, whereas values larger than this began to cover the chin and neck of the person, which introduced new unnecessary features that increase the chance of false classification. Regarding Borders 1 and 2, the borders had to be moved inward to remove any unnecessary details that can cause confusion to the system. The optimal values for Borders 1 and 3 were 20 and 20 pixels, respectively. A value lower than this is futile, because the ears are still covered within the borders, whereas a value higher than this is very bad, because the borders crop parts of the eyes.

\section{Cropping}

One border was left unmodified: the top border. The options here were either to crop the image, including the forehead of the face, or to crop it without the forehead. This choice had to be determined by testing, because the forehead was a point of confusion, and it was not clear whether the forehead contributes to the expression or is just unnecessary.

The system was tested once with the forehead and another time after cropping the forehead from the face. The test was repeated on two datasets: JAFFE and CK+.

Table 1 shows that the forehead is an essential part of the expression and must be included in the face to determine the correct expression. The forehead played an important role in determining expressions such as sad and angry.
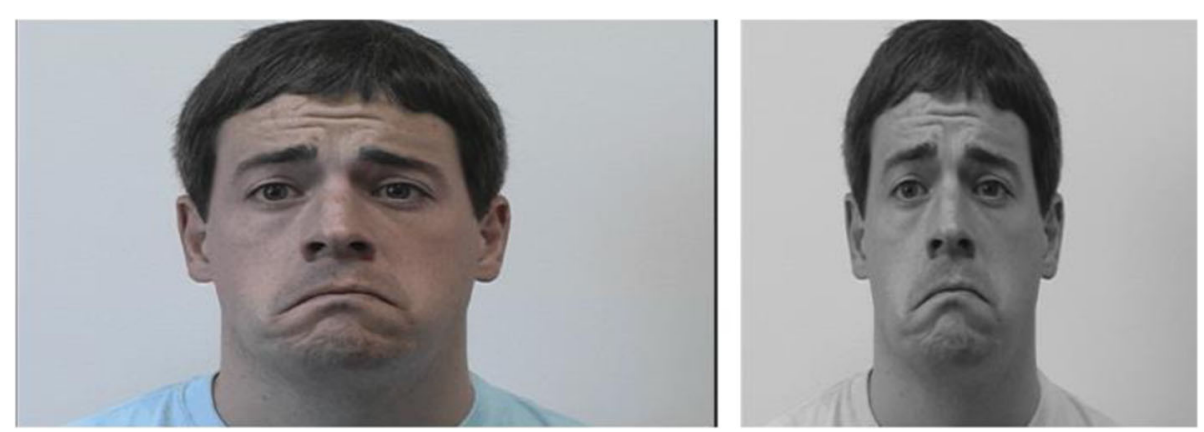

Fig. 5 Grayscale and resizing to $256 \times 256$ 

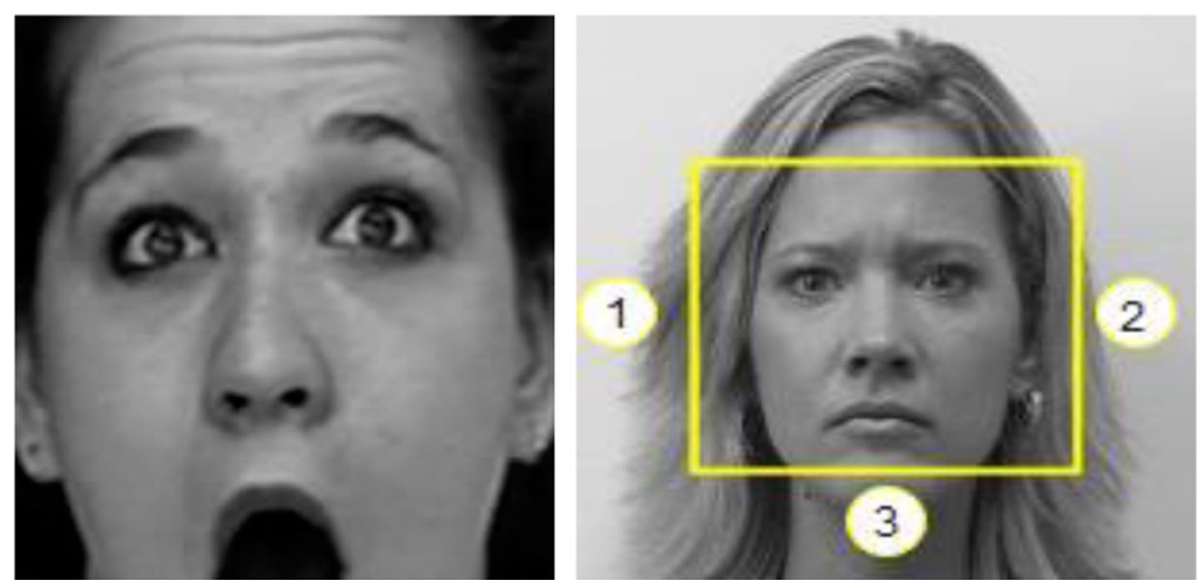

Fig. 6 Viola-Jones face detection with mouth missing (left): Border 3 must cover the whole mouth only, whereas Borders 1 and 2 must cover the face region (right)

\section{Additional resizing}

The last step was to resize the images with the detected face and the adjusted borders. This was done to ensure that the images with the detected faces were all equal in size, because the Viola-Jones algorithm detects faces with different border sizes due to the nature and size of the face and because of the border adjustment step. Therefore, the resizing had to be reimplemented to reach for standard image input.

After applying the border modification to the image, the size of the output image was recorded, and the minimum was noticed. The smallest size that was recorded was $112 \times 92$; therefore, all the images in all the datasets had to be of this size to ensure consistency and ensure the same number of features for all images. Table 1 displays how crucial image preprocessing is, because there was approximately a $2 \%$ and $5 \%$ increase in the accuracy of the Jaffe and CK+ datasets, respectively.

\section{Experimental results and discussion}

Regarding FER, the two most famous and used validation methods to determine accuracy are the leave-oneout validation method and the K-fold validation method.

\section{Leave-one-out validation test}

The leave-one-out validation test is used to determine the accuracy of the system by dividing the dataset into

Table 1 Increase of proposed accuracy method using image preprocessing techniques

\begin{tabular}{lll}
\hline & Datasets & \\
\cline { 2 - 3 } Accuracy & JAFFE & CK+ \\
\hline Before & $94.84 \%$ & $88.66 \%$ \\
After & $96.71 \%$ & $93.29 \%$ \\
\hline
\end{tabular}

two categories: the testing set and the training set. The testing set contains one image, whereas the training set contains the rest of the dataset. The experiment is then repeated until all the images go through the system at least once, thus determining exactly what images are detected incorrectly out of the whole set [34].

For the datasets used, the number of experiments was 213 for JAFFE, 432 for $\mathrm{CK}_{+}$, and 1407 for the RaFD dataset. The results of the leave-one-out validation test on the three datasets are shown in Table 2.

The accuracy of the JAFFE and RaFD datasets was higher than that of the $\mathrm{CK}+$ one, because the numbers of images in each expression in those two datasets are equal, thus causing a fair training, whereas the CK+ dataset has a varying number of images for each expression. Also, the RaFD dataset showed the highest accuracy, because the training sample was extremely large compared with the other two datasets.

\section{K-fold validation test}

The K-fold validation test is one form of the leave-oneout validation test that has several modifications applied to it. In the K-fold validation test, the dataset that has $\mathrm{N}$ images is divided into $\mathrm{K}$ sets. In each run, one of the $\mathrm{K}$ sets is used for testing, and the remaining sets are used for training. The process is repeated until all sets go through the system once. The only difference between the leave-one-out and K-fold validation tests is that the testing sample is larger for the $\mathrm{K}$-fold one, thus decreasing the training set. Also, the randomness of the images being testing is larger, providing more-realistic results.

To validate the results as much as possible, several types of $\mathrm{K}$-fold test were performed. The first test was the 10 -fold validation test. In this test, the dataset was divided into 10 sets, and 10 iterations were applied to the system. The second test was the fivefold validation 
Table 2 Proposed accuracy method using leave-one-out on the three datasets

\begin{tabular}{ll}
\hline Datasets & Leave-one-out \\
\hline JAFFE & $96.71 \%$ \\
CK+ & $93.29 \%$ \\
RaFD & $99.72 \%$ \\
\hline
\end{tabular}

test, in which the dataset was divided into five sets of images. The sets in the fivefold validation test were larger than those in the 10-fold one. The last test was the twofold validation test, in which the dataset was divided into two halves: one for testing and the other for training. The results of the three discussed K-fold tests when applied to the three datasets are shown in Table 3.

The results of the 10-fold test were higher than those of the other two tests, because the training set was larger than that of the five fold and two fold tests. The CK+ accuracy was lower than those of the JAFFE and Radboud datasets, because $\mathrm{CK}+$ is the only dataset that has a different number of images for each expression; therefore, the randomness in this dataset plays a large part in the accuracy achieved. The twofold test had the lowest accuracy of all the K-fold tests, because the system is not being trained enough, thus decreasing accuracy.

\section{Result overview}

The overall results of performing the above-mentioned tests are summarized in Table 4 . Table 4 shows that the model used has a high accuracy, more than $90 \%$, under any test used and using any dataset. The results show that, if the proposed model is trained enough, the accuracy rises, as can be seen from the leave-one-out validation test. The $\mathrm{CK}+$ dataset had the lowest accuracy among the datasets, because images in the $\mathrm{CK}+$ dataset are not well distributed and vary in number.

\section{Comparison}

The last step is to compare the results obtained with results reported in previous work. Table 5 shows the results obtained by several researchers who developed FER systems. Each decided to implement a certain type of validation test different from the rest. In the last column of Table 5, the type of validation test performed is given.

Regarding the JAFFE database, the leave-one-out test result obtained surpassed the LDA + SVM [4] model by

Table 3 Proposed accuracy method using K-fold method

\begin{tabular}{llll}
\hline & Folds & \\
\cline { 2 - 4 } Datasets & 10 & 5 & 2 \\
\hline JAFFE & $98.10 \%$ & $97.62 \%$ & $90.10 \%$ \\
CK+ & $94.42 \%$ & $93.49 \%$ & $90.00 \%$ \\
RaFD & $95.14 \%$ & $95.10 \%$ & $94.88 \%$ \\
\hline
\end{tabular}

Table 4 Proposed accuracy methods using different folds

\begin{tabular}{lllll}
\hline Datasets & \multicolumn{3}{l}{ Methods } \\
\cline { 2 - 5 } & Leave-one-out & 10 folds & 5 folds & 2 folds \\
\hline JAFFE & $96.71 \%$ & $98.10 \%$ & $97.62 \%$ & $90.10 \%$ \\
CK+ & $93.29 \%$ & $94.42 \%$ & $93.49 \%$ & $90.00 \%$ \\
RaFD & $99.72 \%$ & $95.14 \%$ & $95.10 \%$ & $94.88 \%$ \\
\hline
\end{tabular}

$1 \%$, whereas the model that uses the same extraction and classification techniques with HOG and SVM [5] produced results that were less than those of the proposed model by $2.41 \%$. The results of the K-fold tests also surpassed those of the previous models, especially the 10-fold model that had a huge accuracy difference of $5.70 \%$.

The CK+ dataset also surpassed the previous models that used the same extraction and classification technique and could compete and surpass the performance of a deep neutral network [7], which is considered optimal in comparison with machine learning.

Regarding the RaFD dataset, although it is not very popular in the machine-learning domain, it was possible to compare it with a work that used the 10-fold test and was able to surpass it by more than $1 \%$.

\section{Conclusions}

An effective method of addressing the FER problem was proposed. Several steps were performed at the image preprocessing stage to ensure that the features attained from the face detected have a meaningful and proper contribution to the classification stage. The extra features at the side of the face (around the ears) and the ones at the bottom of the mouth (around the chin and

Table 5 Proposed accuracy method compared with other techniques

\begin{tabular}{lllll}
\hline Datasets & Method & $\begin{array}{l}\text { Classification } \\
\text { rate }\end{array}$ & $\begin{array}{l}\text { Proposed } \\
\text { method }\end{array}$ & Validation test \\
\hline JAFFE & $\begin{array}{l}\text { Patch-based } \\
\text { Gabor [5] }\end{array}$ & $92.30 \%$ & $96.71 \%$ & Leave-one-out \\
& HOG+SVM [5] & $94.30 \%$ & & \\
& LDA + SVM [4] & $95.71 \%$ & & \\
& Boosted LBP + & $79.80 \%$ & $98.10 \%$ & 10-folds \\
& SVM [3] & & & \\
& LBP pyramid + & $91.36 \%$ & & \\
& SVM [1] & & & \\
& LBP + Gabor & $92.38 \%$ & & 5-folds \\
& filter [1] & & & Leave-one-out \\
& LBP + HOG + & $87.60 \%$ & $97.62 \%$ & 5-fold \\
PCA + SVM [6] & & & 10-fold \\
\hline CK+ & HOG+SVM [5] & $88.70 \%$ & $93.29 \%$ & \\
& DNN [7] & $93.20 \%$ & $93.49 \%$ & \\
RaFD & HOG+SVM [20] & $98.50 \%$ & $99.72 \%$ &
\end{tabular}


neck) proved not to contribute any positive or useful information to the classification stage. Thus, it was necessary to crop them. In addition, it was essential for the mouth to be detected as a whole and not be cropped. Facial expressions were created as a result of muscle movements on the face. These subtle movements were detected by the HOG features, because HOG is sensitive to the shape of objects. The features attained are then used to train a network of binary linear SVMs. This network consists of three stages where the primary and first stage is made up of 21 SVMs that are all the binary combinations of the seven expressions. If one expression is dominant, then the first stage suffices. If two are dominant, then the second stage is used, and, if three are dominant, then the third stage is used. These multilevel stages reduce, as much as possible, the possibility of experiencing an error. Experimental results on three databases - JAFFE, CK+, and RaFD - show that the proposed system is competitive and has better performance compared with previous research results. Two different validation methods were used: the leave-one-out and $\mathrm{K}$-fold tests. The percentages obtained from the leave-one-out test were $96.71 \%, 93.29 \%$, and $99.72 \%$ for the JAFFE, CK+, and RaFD datasets, respectively. However, the percentages obtained from the 10-fold test (the most common fold test) were $98.10 \%, 94.42 \%$, and $95.14 \%$, respectively.

\section{Abbreviations}

CK+: The Extended Cohn-Kanade Dataset; FER: Facial Expression Recognition; HOG: Histogram of Oriented Gradients; JAFFE: Japanese Female Facial Expressions; LBP: Local Binary Patterns; RaFD: Radboud Facial dataset; SVMs: Support Vector Machines

\section{Acknowledgements}

We thank our intelligent team.

\section{Authors' contributions}

Results show that our proposed system is very competitive and has better performance in relation to other works. All authors read and approved the final manuscript.

\section{Authors' information \\ Issam Dagher finished his MS in electrical engineering degree in 1994 from Florida International University, Miami, USA. He finished his PhD in 1997 from Univesity of Central Florida, Orlando, USA. He is now an associate professor at the University of Balamand, Lebanon. His area of interests are pattern recognition, neural networks, artificial intelligence, computer vision. He published many papers on these topics. \\ Elio Dahdah and Morshed Elshakik finished their MS in computer engineering in 2017 from the University of Balamand. Their area of interests are machine learning and artificial intelligence.}

\section{Funding}

No.

\section{Availability of data and materials} Upon request.

Ethics approval and consent to participate I consent.

\section{Consent for publication}

Photos from Figures 3, 4, 5, and 6 are obtained from the JAFFE, CK+ and RaFD public databases.

\section{Competing interests}

The authors declare that they have no competing interests.

Received: 25 September 2019 Accepted: 18 November 2019

Published online: 16 December 2019

\section{References}

1. Jaffar MA (2017) Facial expression recognition using hybrid texture features based ensemble classifier. Int J Adv Comput Sci Appl 8(6). https://doi.org/ 10.14569/IJACSA.2017.080660

2. Poria S, Mondal A, Mukhopadhyay P (2015) Evaluation of the intricacies of emotional facial expression of psychiatric patients using computational models. In: Mandal MK, Awasthi A (eds) Understanding facial expressions in communication. Springer, New Delhi, pp 199-226. https://doi.org/10.1007/ 978-81-322-1934-7_10

3. Shan CF, Gong SG, McOwan PW (2009) Facial expression recognition based on local binary patterns: a comprehensive study. Image Vis Comput 27(6): 803-816. https://doi.org/10.1016/j.imavis.2008.08.005

4. Shih FY, Chuang CF, Wang PSP (2008) Performance comparisons of facial expression recognition in JAFFE database. Int J Pattern Recognit Artif Intell 22(3):445-459. https://doi.org/10.1142/S0218001408006284

5. Chen JK, Chen ZH, Chi ZR, Fu H (2014) Facial expression recognition based on facial components detection and hog features. In: Abstracts of scientific cooperations international workshops on electrical and computer engineering subfields, Koc University, Istanbul, Turkey, 22-23 August 2014, pp 64-69

6. Liu YP, Li YB, Ma X, Song R (2017) Facial expression recognition with fusion features extracted from salient facial areas. Sensors 17(4):712

7. Mollahosseini A, Chan D, Mahoor MH (2016) Going deeper in facial expression recognition using deep neural networks. In: Abstracts proceedings of 2016 IEEE winter conference on applications of computer vision. IEEE, Lake Placid, 7-10 March 2016, pp 1-10. https:/doi.org/10.1109/WACV.2016.7477450

8. Carcagnì $P$, Del Coco M, Leo M, Distante C (2015) Facial expression recognition and histograms of oriented gradients: a comprehensive study. SpringerPlus 4:645. https://doi.org/10.1186/s40064-015-1427-3

9. Savran A, Sankur B (2017) Non-rigid registration based model-free 3D facial expression recognition. Comput Vis Image Underst 162:146-165. https://doi. org/10.1016/j.cviu.2017.07.005

10. Sharma K, Rameshan R (2017) Dictionary based approach for facial expression recognition from static images. In: Mukherjee S, Mukherjee S, Mukherjee DP, Sivaswamy J, Awate S, Setlur S et al (eds) Computer vision, graphics, and image processing. ICVGIP 2016 satellite workshops, December 2016. Lecture notes in computer science (Lecture notes in artificial intelligence), vol 10484. Springer, Cham, pp 39-49. https://doi.org/10.1007/ 978-3-319-68124-5_4

11. Bashyal S, Venayagamoorthy GK (2008) Recognition of facial expressions using Gabor wavelets and learning vector quantization. Eng Appl Artif Intell 21(7):1056-1064. https://doi.org/10.1016/j.engappai.2007.11.010

12. Biswas S, Sil J (2015) An efficient expression recognition method using contourlet transform. In: Abstracts of the 2 nd international conference on perception and machine intelligence. ACM, Kolkata, West Bengal, 26-27 February, 2015, pp 167-174. https://doi.org/10.1145/2708463.2709036

13. Gong BQ, Wang YM, Liu JZ, Tang XO (2009) Automatic facial expression recognition on a single $3 \mathrm{D}$ face by exploring shape deformation. In: Abstracts of the 17th ACM international conference on multimedia. ACM, Beijing, 19-24 October 2009, pp 569-572

14. Tsai HH, Chang YC (2018) Facial expression recognition using a combination of multiple facial features and support vector machine. Soft Comput 22(13):4389-4405. https://doi.org/10.1007/s00500-017-2634-3

15. Clawson K, Delicato LS, Bowerman C (2018) Human centric facial expression recognition. In: Abstracts of the 32nd international BCS human computer interaction conference, electronic workshops in computing, Belfast, UK, 4-6 July 2018, pp 1-12. https://doi.org/10.14236/ewic/HCI2018.44

16. Cossetin MJ, Nievola JC, Koerich AL (2016) Facial expression recognition using a pairwise feature selection and classification approach. In: Abstracts of 2016 international joint conference on neural networks. IEEE, Vancouver, 24-29 July 2016, pp 5149-5155. https://doi.org/10.1109/IJCNN.2016.7727879 
17. Cui RX, Liu MY, Liu MH (2016) Facial expression recognition based on ensemble of mulitple CNNs. In: You ZS, Zhou J, Wang YH, Sun ZN, Shan SG, Zheng WS et al (eds) Biometric recognition. 11th Chinese conference, CCBR 2016, October 2016. Lecture notes in computer science (Lecture notes in artificial intelligence), vol 9967. Springer, Cham, pp 511-518. https:/doi.org/10.1007/978-3-319-46654-5_56

18. Dahmane M, Meunier J (2014) Prototype-based modeling for facial expression analysis. IEEE Trans Multimed 16(6):1574-1584. https://doi.org/10. 1109/TMM.2014.2321113

19. Uçar A, Demir Y, Güzeliş C (2014) A new facial expression recognition based on curvelet transform and online sequential extreme learning machine initialized with spherical clustering. Neural Comput Appl 27(1):131-142. https://doi.org/10.1007/s00521-014-1569-1

20. Ekman P, Friesen WV (2003) Unmasking the face: a guide to recognizing emotions from facial clues. Malor Books, Cambridge

21. Vezzetti E, Marcolin F, Tornincasa S, Ulrich L, Dagnes N (2017) 3D geometry-based automatic landmark localization in presence of facial occlusions. Multimed Tools Appl 77(11):14177-14205. https:/doi.org/10.1007/s11042-017-5025-y

22. Gao YS, Leung MKH, Hui SC, Tananda MW (2003) Facial expression recognition from line-based caricatures. IEEE Trans Syst Man Cybern A Syst Hum 33(3):407-412. https://doi.org/10.1109/TSMCA.2003.817057

23. Guo M, Hou XH, Ma YT, Wu XJ (2017) Facial expression recognition using ELBP based on covariance matrix transform in KLT. Multimed Tools Appl 76(2):2995-3010. https://doi.org/10.1007/s11042-016-3282-9

24. Soyel H, Demirel H (2007) Facial expression recognition using 3D facial feature distances. In: Kamel M, Campilho A (eds) Image analysis and recognition. 4th international conference, august 2007. Lecture notes in computer science (lecture notes in artificial intelligence), vol 4633. Springer, Heidelberg, pp 831-838. https://doi.org/10.1007/978-3-540-74260-9_74

25. Happy SL, Routray A (2015) Automatic facial expression recognition using features of salient facial patches. IEEE Trans Affect Comput 6(1):1-12. https:// doi.org/10.1109/TAFFC.2014.2386334

26. Hegde GP, Seetha M, Hegde N (2016) Kernel locality preserving symmetrical weighted fisher discriminant analysis based subspace approach for expression recognition. Eng Sci Technol Int J 19(3):1321-1333. https://doi. org/10.1016/j.jestch.2016.03.005

27. Hernandez-matamoros A, Bonarini A, Escamilla-Hernandez E, NakanoMiyatake M, Perez-Meana H (2015) A facial expression recognition with automatic segmentation of face regions. In: Fujita H, Guizzi G (eds) Intelligent software methodologies, tools and techniques. 14th international conference, September 2015. Lecture notes in computer science (Lecture notes in artificial intelligence), vol 532. Springer, Cham, pp 529-540. https:// doi.org/10.1016/j.knosys.2016.07.011

28. Li HB, Sun J, Xu ZB, Chen LM (2017) Multimodal 2D + 3D facial expression recognition with deep fusion convolutional neural network. IEEE Trans Multimed 19(12):2816-2831. https://doi.org/10.1109/TMM.2017.2713408

29. Islam DI, SRN A, Datta A (2018) Facial expression recognition using 2DPCA on segmented images. In: Bhattacharyya S, Chaki N, Konar D, Chakraborty U, Singh $C T$ (eds) Advanced computational and communication paradigms. Proceedings of international conference on ICACCP 2017. Advances in intelligent systems and computing, vol 706. Springer, Singapore, pp 289297. https://doi.org/10.1007/978-981-10-8237-5_28

30. Jain S, Durgesh M, Ramesh T (2016) Facial expression recognition using variants of LBP and classifier fusion. In: Satapathy SC, Joshi A, Modi N, Pathak N (eds) Proceedings of international conference on ICT for sustainable development. Advances in intelligent systems and computing, vol 42. Springer, Heidelberg, pp 725-732. https:/doi.org/10.1007/978-981-10-0129-1_75

31. Lyons M, Kamachi M, Gyoba J (1998) The Japanese female facial expression (Jaffe) database. https://zenodo.org/record/3451524\#.XbvRmfk6s7M. Accessed: 19 April 2019

32. Lucey P, Cohn JF, Kanade T, Saragih J, Ambadar Z, Matthews I (2010) The extended cohn-kanade dataset (CK+): a complete dataset for action unit and emotionspecified expression. In: Abstracts of 2010 IEEE computer society conference on computer vision and pattern recognition-workshops. IEEE, San Francisco, 13-18 June 2010, pp 94-101. https://doi.org/10.1109/CVPRW.2010.5543262

33. Langner O, Dotsch R, Bijlstra G, Wigboldus DHJ, Hawk ST, van Knippenberg A (2010) Presentation and validation of the Radboud faces database. Cognit Emot 24(8):1377-1388. https://doi.org/10.1080/02699930903485076

34. Kumar A (2018) Machine learning: validation techniques. https://dzone.com/ articles/machine-learning-validation-techniques. . Accessed: 19 April 2019

\section{Publisher's Note}

Springer Nature remains neutral with regard to jurisdictional claims in published maps and institutional affiliations.

\section{Submit your manuscript to a SpringerOpen ${ }^{\circ}$ journal and benefit from:}

- Convenient online submission

- Rigorous peer review

- Open access: articles freely available online

High visibility within the field

- Retaining the copyright to your article

Submit your next manuscript at $\boldsymbol{\nabla}$ springeropen.com 